\title{
Research of Partial Discharge Monitoring for Switchgear Cabinet Based on LSSVM
}

\author{
Shai Gen HAN ${ }^{1, a}$, Shi Lei GUAN ${ }^{1}$, Yan WU ${ }^{1}$, Zhi Xin BO ${ }^{1}$, Yan DING ${ }^{1}$, Li An CHEN ${ }^{2}$ \\ ${ }^{1}$ China Electric Power Research Institute, Beijing 100192, China \\ ${ }^{2}$ Xiamen University of Technology, Xiamen 361024, China
}

\begin{abstract}
Now, there are many influencing factors of partial discharge(PD) for switchgear cabinet, but very few methods used for switchgear cabinet monitoring. In this paper, Partial discharge of switchgear cabinet is identified based on Least Square SVM (LSSVM) used for comprehensive evaluation of switchgear cabinet. Partial discharge ultrasonic signal of time and frequency domain are gave to get two stable characteristic bands in MATLAB tool. It provides a theoretical basis for partial discharge monitoring to ensure proper and efficient operation of the switchgear cabinet.
\end{abstract}

Key words: Partial Discharge; Switchgear Cabinet; LSSVM; Ultrasonic Signal.

\section{Introduction}

The insulating materials in high voltage switchgear cabinet are vulnerable to the effects of chemical, mechanical, electrical, thermal and other factors. The defect of insulating materials can lead to partial discharge and prompt the cumulative charge and thus causes insulation failures, which accounts for more than $50 \%$ of electricity equipment failures. The insulation structures, materials and workmanship determine the reliability of electrical equipments' reliability. Papers ${ }^{[1-5]}$ research the phenomenon of partial discharge, but the results are not complete, and the test is not very good. Papers ${ }^{[6-8]}$ use ultrasonic method to test arc fault, but the dynamic performance and accuracy rate are difficult to meet the requirements, and the calculation algorithms are very complicated. Therefore, studying one new method which is suitable for high voltage switchgear discharge monitoring is particularly meaningful ${ }^{[1-3]}$.

\section{Factors affecting the partial discharge in switchgear}

In the electric field, the gas gap in the vicinity of electrical conductors or in other regions is broken down, and the discharge phenomenon is called partial discharge. When partial discharge occurs, a variety of high-energy charged particles, such as electrons, positive and negative ions, etc., in the electric field strength of traction, these charged particles strike the walls of the air gap to the media, resulting in extremely high temperatures which

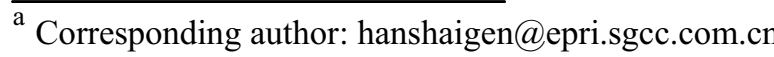

causes the insulation material damage. At present, the mechanical, electrical faults high voltage switchgear are:

(1) Insulation malfunction. The main reason for this failure is that the internal insulation material is brokendown. This is the main fault of the switchgear.

(2) Refuse to move or malfunction failure. The main reasons for this failure are mechanical transmission, electrical control and auxiliary circuits. This is more fault occurred in the switchgear.

(3) Malfunction of breaking and closing. this is caused by that the breaking and closing force of the circuit breaker is insufficient, and gas leakage of arc extinguish chamber, what is the common fault of switchgear.

(4) Malfunctions caused by external forces.

\section{LSSVM's application in detecting the partial discharge}

With the research of support vector machine algorithm ${ }^{[4-9]}$, it is found that the method of using standard support vector in partial discharge detection has many limitations, and many scholars have put forward the improved algorithm of support vector machine. Among them, least squares support vector machine algorithm, Bias support vector machine, Lagrange support vector machines, simplified support vector machines are widely used. When using the least square support vector machine to train samples, it only needs to solve a linear equation group, which greatly improves the computational speed 
and training efficiency, and thus meet the requirements of system modeling and identification.

Least squares support vector machine(LSSVM) method converts solving nonlinear equations to a set of linear algebraic equations, and converts the uncertain solution into certain solution, and as well as converts the standard support vector machine constraints by the inequations into the equations, thus greatly reducing the training time. Experiments show that the generalization performance of LSSVM is higher than that of the standard SVM.

Partial discharge detection is a process of two classification, and according to the criteria of SVM conditions, the constraint conditions of LSSVM can be equivalent to:

$$
\mathrm{y}_{i}\left[w^{T} \phi\left(x_{i}\right)+\mathrm{b}\right]=1-\xi_{i}, \quad i=1,2,3,4 \ldots \ldots, N
$$

The objective function of LSSVM is:

$$
\begin{aligned}
& \left\{\begin{array}{c}
\min J(w, \xi)=\frac{1}{2} w^{T} w+\frac{c}{2} \sum_{i=1}^{N} \xi^{2} \\
\text { s.t. } \mathrm{y}_{i}\left[w^{T} \phi\left(x_{i}\right)+\mathrm{b}\right]=1-\xi_{i}, i=1,2,3,4 \ldots . ., N
\end{array}\right. \\
& \max Q(\mathrm{w}, b, a, \xi)=J(w, \xi)-\sum_{i=1}^{N} a_{i}\left\{y_{i}\left[w^{T} \phi\left(x_{i}\right)+\mathrm{b}\right]-1+\xi_{i}\right\}
\end{aligned}
$$

The equation (3) is the dual Lagrange polynomials of equation (2), $a_{i}$ is Lagrange multiplier. At the same time we calculate the partial derivation of $w 、 b 、 a 、 \xi$ and then equation (4) is derived.

$$
\left\{\begin{array}{c}
\frac{\partial Q}{\partial w}=0 \Rightarrow w=\sum_{i=1}^{N} a_{i} y_{i} \phi\left(x_{i}\right) \\
\frac{\partial Q}{\partial b}=0 \Rightarrow \sum_{i=1}^{N} a_{i} y_{i}=0 \\
\left.\frac{\partial Q}{\partial a}=0 \Rightarrow y_{i}\left[w^{T} \phi\left(x_{i}\right)+b\right]-1+\xi_{i}\right\}=0 \\
\frac{\partial Q}{\partial \xi}=0 \Rightarrow a_{i}=c \xi_{i}
\end{array}\right.
$$

where $a_{i}$ and the training error $\xi_{i}$ are linear proportional relation, and the equation can be obtained by being simplified as:

$$
\sum_{i=1}^{N} \sum_{j=1}^{M} a_{i}\left[y_{i} y_{j} \phi^{T}\left(x_{i}\right) \phi\left(x_{j}\right)+\frac{\delta_{i j}}{c}\right]+b y_{j}=1
$$

Let $\Omega_{i j}=y_{i} y_{i} \phi^{T}\left(x_{i}\right) \phi\left(x_{j}\right)+\frac{\delta_{i j}}{c} \quad, \quad$ where $\delta_{i j}=\left\{\begin{array}{ll}1 & i=j \\ 0 & i \neq j\end{array}\right.$. According to Mercer's theory we can get $\phi^{T}\left(x_{i}\right) \phi\left(x_{j}\right)=\psi\left(x_{i}, x_{j}\right)$, we can get linear equations equation (6) of $a$ and $b$ with the third equation in equations (4) and equation (5).

$$
\left[\begin{array}{ll}
\Omega & y \\
y^{T} & 0
\end{array}\right]\left[\begin{array}{l}
a \\
b
\end{array}\right]=\left[\begin{array}{l}
1 \\
0
\end{array}\right]
$$

In equation (6), $\Omega$ is a Semi-definite symmetric matrix whose diagonal elements are $1 / \mathrm{C}$, and we can derive equation (7):

$$
\left\{\begin{array}{l}
a=\Omega^{-1}(1-y b) \\
b=\left(y^{T} \Omega^{-1} y\right)^{-1} y^{T} \Omega^{-1} 1
\end{array}\right.
$$

Set up the two classification mode by the least squares method $^{[10-13]}$, and choose the best characteristic quantities and parameters based on the classification to improve the promotion and viability, which was divided into the following four steps:

(1) Data normalization. When partial discharge occurs, the ultrasonic frequencies is located in: (a) $20 \sim 40 \mathrm{kHz}$, (b) $80 \sim 140 \mathrm{kHz}$. Through the signal conditioning circuit, the integrated value of these two frequencies of ultrasound is limited to $0-1$.

(2) Establishment of SVM's sample database. By simulating six kinds of working conditions under the discharge model, to create the sample database, in order to achieve fully optimized results. supposing the threshold parameter is $\mathrm{H}$, and the tag value is $\mathrm{Y}$, when $\mathrm{H}$ $\geqslant 90 \mathrm{pC}$, it is marked as partial discharge phenomena, and $\mathrm{Y}=1$; when $\mathrm{H}<90 \mathrm{pC}$, it is marked no partial discharge, $\mathrm{Y}=-1$.

(3)Train and test samples. Training samples contain various partial discharge conditions, and we train a total of 150 sets of data samples, where 50 sets of data are used to test samples, 100 sets of data are used to train the samples. Table 1 shows part of the PD data.

Table 1. Part PD data

\begin{tabular}{|c|c|c|c|}
\hline $\begin{array}{c}\text { Sample } \\
\text { number }\end{array}$ & $\begin{array}{c}\text { Feature vector } \\
\text { S1 }\end{array}$ & $\begin{array}{c}\text { Feature vector } \\
\text { S2 }\end{array}$ & Output \\
\hline 1 & 0.323457 & 0.143148 & -1 \\
\hline 2 & 0.321687 & 0.144001 & -1 \\
\hline 3 & 0.384538 & 0.174869 & 1 \\
\hline$\ldots$ & $\ldots$ & $\ldots$ & $\ldots$ \\
\hline 149 & 0.326145 & 0.156784 & -1 \\
\hline 150 & 0.381357 & 0.246034 & 1 \\
\hline
\end{tabular}

(4) Select kernel function $\delta^{2}$ and penalty factor $c$.In this work, grid optimization method is used. And we choose RBF function classification kernel function, and cross-validation is used to calculate the mean square error. The horizontal axis is set to the penalty factor $c$, and the vertical axis is set to kernel function $\sigma 2$. After verification, the minimum MSE value set is derived, which are the optimal values. This strategy is fast, easy and has features of a high calculation precision. And using this method we can successfully get the best global 
solution. After calculation and analysis, $c=20$, $\delta^{2}=0.005$ is derived.

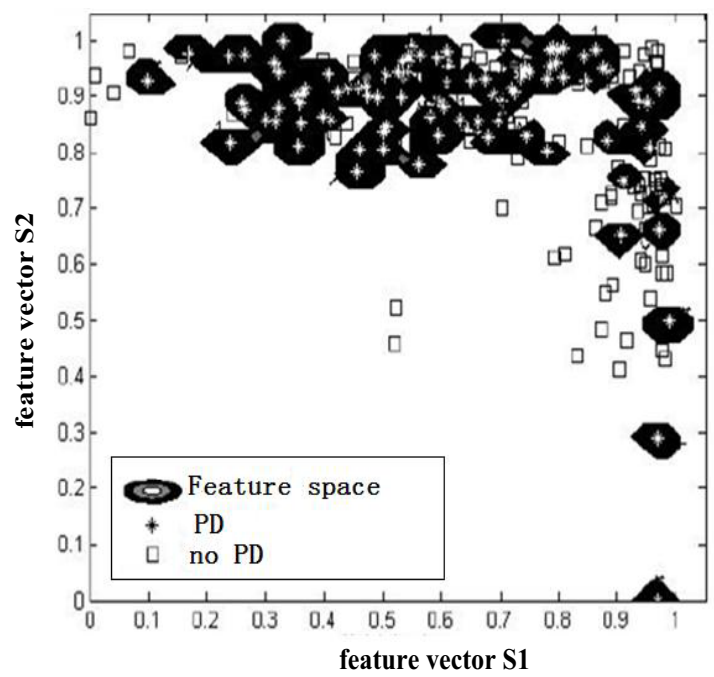

Figure 1. Recognition PD pattern rendering

As shown in Figure 1, " $\square$ " represents the samples that there are no PD existence, "*" represents the samples that there are PD existence. When there is no partial discharge the samples will be scattered outside the feature space, and samples that fall on the boundary are the feature vectors, and when there is no partial discharge the samples will fall within the feature space, the feature vector consists of the classification hyperplane, and samples without partial discharge can be apart from the sample. LSSVM classification model has a strong learning ability and generalization. This model can successfully identify partial discharge phenomenon.

\section{Experimental platform for PD}

Figure 2 is the sketch of experimental platform for PD. This experimental platform is consist of the transformer control box, water resistance, shielding network, metal plate frame, insulating rubber mat, step-up transformer and other necessary equipments. The transformer's capacity is $50 \mathrm{kVA}$, The adjustment range is $0 \sim 400 \mathrm{~V}$, and the rated current is $125 \mathrm{~A}$, the transformer model is YDWB-50kVA/50kV, the voltmeter shows the setting voltage. Water resistance is used to buck and restrict the current, the gap adjusting wheel is composed of insulating rod, electrode, adjusting shaft, spinner wheel device. Control the discharge distance by adjusting the hand wheel through the rotary gap.

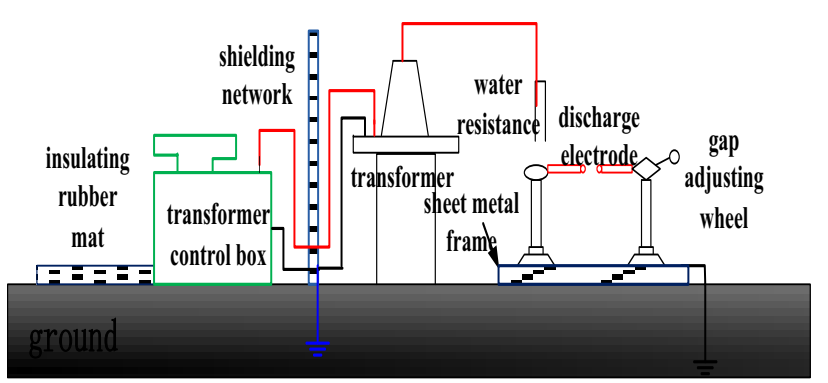

Figure 2. Sketch of experimental platform for PD

Use the experimental platform to simulate the six kinds of partial discharge, and each kind PD is tested for 8 times. DSP control system can accurately check the arc fault, and it can send warning signals and give trip command simultaneously. Actuator can do protected action, reliability, speed instantaneous trip, to meet the accuracy requirements.

MATLAB is used to analyze ultrasonic data processing. In this work, the frequency response range of ultrasonic sensor is $22 \mathrm{kHz} 220 \mathrm{kHz}$, and the sampling frequency is $2 \mathrm{MHz}$, which is in line with Shannon sampling theorem.

Fig. 3 shows the time-domain characteristics of the six discharge models. The distance between electrodes of needle-stick model is $25 \mathrm{~mm}$, and the distance between electrodes of the other five situations is $60 \mathrm{~mm}$. Six discharge models are obtained by the $\mathrm{z}$ Butterworth bandpass filter in the frequency of $22 \mathrm{kHz} \sim 220 \mathrm{kHz}$ and the mean filter is used to get the ultrasonic time-domain waveform.
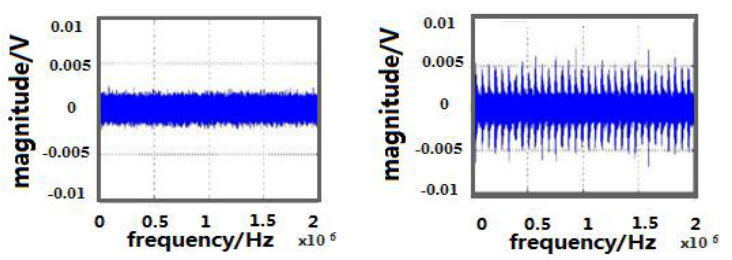

(a) pin-plate
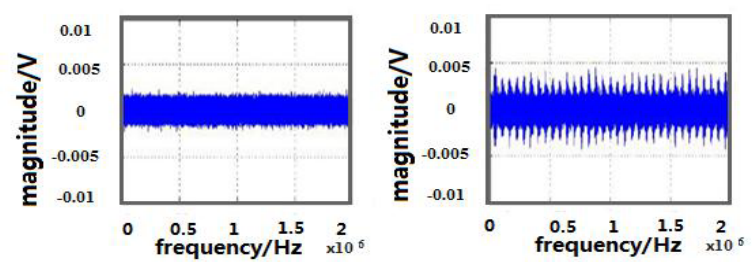

(b) pin-ball
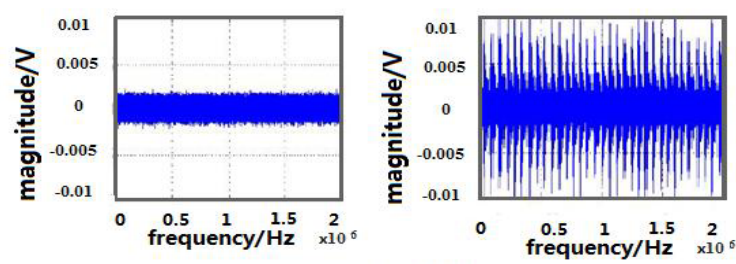

(c) pin-stick 


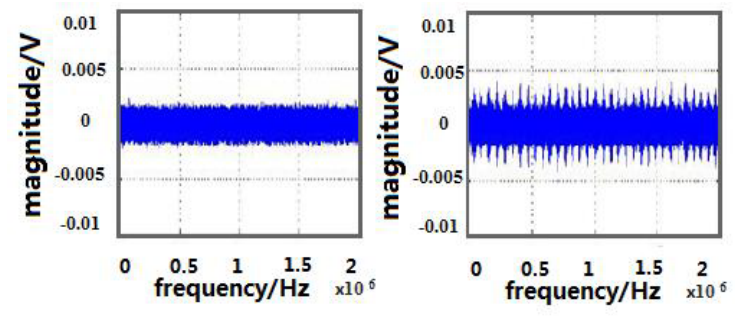

(d) pin-plate
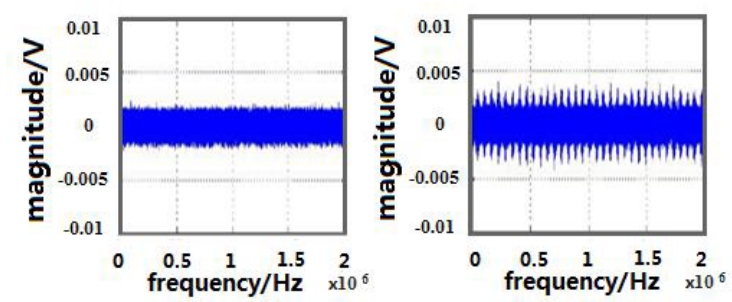

(e) stick-ball

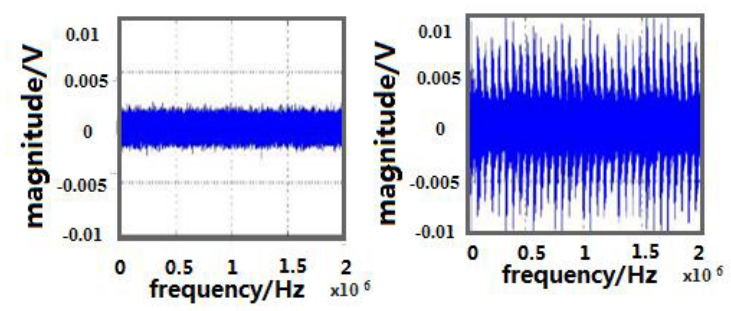

(f) suspension

Figure 3. Six diagrams of discharge time-domain model

As shown in Figure 3, when there is no partial discharge, the ultrasonic wave signal is very smooth and has no obvious peaks. It's amplitude fluctuation is in $\pm 2.8 \mathrm{mV}$. When partial discharge occurs, the waveform has obvious burr and the ultrasonic signal's fluctuation increases to $\pm 5.0 \mathrm{mV}$. Among them, ultrasonic signal amplitude of needle-stick model and suspended model can even reach $\pm 10.0 \mathrm{mV}$, which is about three times larger than that of no PD occurring.
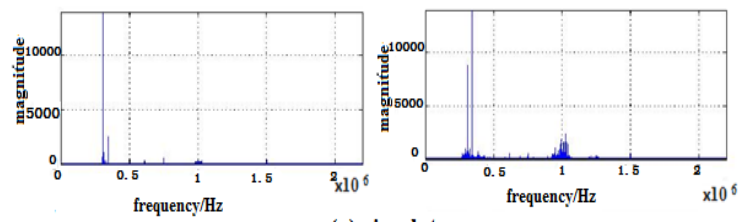

(a) pin-plate
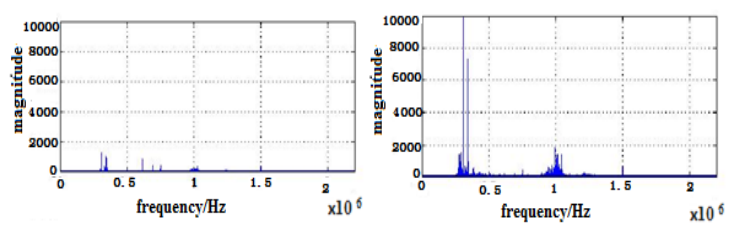

(b) pin-ball
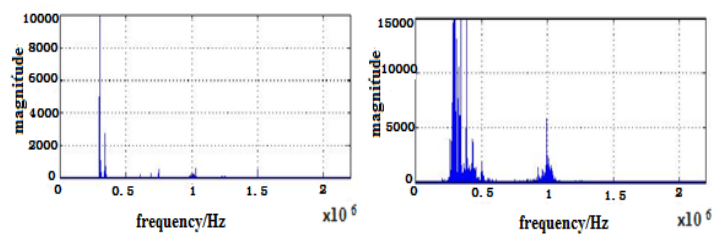

(c) pin-stick
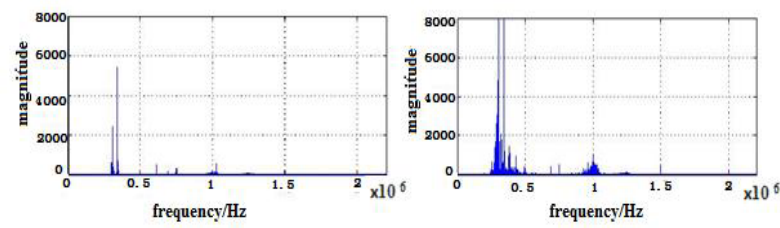

(d) pin-ball
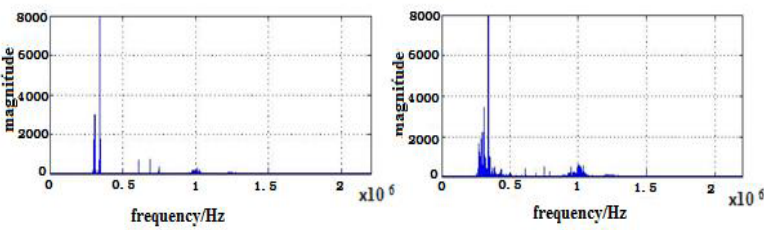

(e) stick-ball
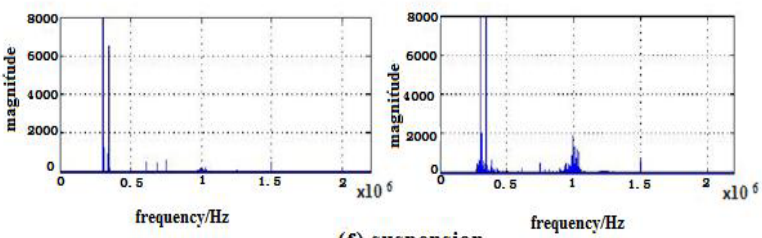

(f) suspension

Figure 4. Six kinds of discharge model spectrums

As shown in Figure 4, when there is partial discharge, ultrasonic signal has been significantly enhanced, the signals have a very intensive frequency distribution. Its frequency energy band focused on the two frequency bands: (a) $20 \sim 40 \mathrm{kHz}$, (b) $80 \mathrm{k} \sim 140 \mathrm{kHz}$. It is found that needle-stick model has the power spectrum value of the maximum discharge. In the absence of partial discharge ultrasonic frequency waveforms concentrated around $30 \mathrm{kHz}$, and the frequency with relatively sparse distribution, the amplitude is relatively small. Since the electrode is relatively sharp. The needle-stick model suffers severe electric field distortion, resulting in a more intense partial discharge phenomena.

\section{Conclusion}

We use partial discharge experimental platform to analyze the partial discharge mechanism in switchgear cabinet. The strategy using LSSVM is proposed to detect partial discharge of switchgear according to causes and mechanism of partial discharge. Six kinds of PD are simulated, then we use MATLAB to do a great deal of experiments for different discharge models. According to time-domain waveforms and frequency-domain waveforms, the essence of the phenomenon of partial discharge is captured, and it provides an experimental basis for the characteristic values of partial discharge. 


\section{References}

[1] D Hao-yin, T. Neng-ling, C. Xin-qi, G. Jian-zhong, F. Chun-ju. J. Electr. Power Sci. and Technol., 25, 64(2010) [2] W. Quan, C. Hong-song, ZH. Han-dong, Y. Da-wei. J. Gun Launch \& Control, 3, 59(2010)

[3] Z. Wen-qi, X. Li-yun, ZH. Li-lin. J. Electr. Power Sci. and Technol., 25, 32(2010)

[4] H. Wei-tao.J. Electr. Power Sci. and Technol., 27, 57(2012)

[5] M. Peiqing, L. Xiuwei, H. Yue, Sh. Gehao, J. Xiuchen. PEAM. IEEE,1(2012)

[6]R. Widodo; Z. Ming-Xiao; Suwarno; Zh.Guan-Jun. ICEEI, 181(2015)
[7] M. Kida, T. Kawashima, Y. Murakami, M. Nagao. CEIDP, 322(2015)

[8]T.S. Sidhu, M.S. Sachdev, G.S. Sagoo . Dev. in Power Syst. Prot., 157(2010)

[9] L. De-jun, SH. Wei, G. Zhi-qiang. High-voltage E.A., 45, 99(2009)

[10] K. Siderakis, E. Pyrgioti, E. Thalassinakis, I. Vitellas. IEEE Trans. Dielectr. E.I. 18, 122(2011)

[11] W. Zhang-jian, L. Cheng-rong, Q. Bo, H. Zhen, G. Bibo. Mod. Electr. Power, 27, 31(2010)

[12] Z. Lei. Yunnan Electric Power, 39, 49(2011)

[13] Z.Li-lin. SHH Electr. Power, 23, 50(2011) 